\title{
UV Spectrophotometer Based AI Techniques for Remnant Life Estimation of Power Transformers
}

\author{
Satyaprakash Ram ${ }^{1}$, Dr. A.K. Chandel ${ }^{2}$, Gurmeet Singh $^{3}$, Mithun Mondal ${ }^{4}$ \\ 1,2,3,4 (EED, National Institute of Technology, Hamirpur (H.P), India)
}

\begin{abstract}
Power transformers are critical equipment in the electrical power system. Diagnostic and condition monitoring is very important for testing of power transformer oil in order to find the remnant life and has to be done carefully. Mineral oil in transformer serves two purposes the insulating as well as cooling and it is inseparable material of dielectric insulation system. The oil gets contaminated mainly due to ageing. The contaminant which changes the oils chemical and physical properties are moisture, acids, metal particles, sludge, and other compounds which are present in cellulose and due to aging insulation of the cellulose. UVSpectrophotometer response is a nonintrusive test used to determine the transformer integrity and the response can be measured instantly with relatively cheap equipment and there is no need for an expert person. The present paper introduces two approaches of AI techniques they viz. Fuzzy logic and Artificial neural network $(A N N)$ to estimate the relationship between dissolve decay content and UV spectrophotometer response of transformer oil method to determine the remnant life of the transformer oil. These two methods use the UV spectrophotometer bandwidth and absorbance values of the transformer oil as the inputs which are in service at several locations.
\end{abstract}

Keywords: Artificial Neural network (ANN), Dissolve Decay Contents, Fuzzy logic, Transformer oil, UV Spectrophotometer.

\section{Introduction}

Power transformer is extremely important electrical equipment in the power system as well as in industries. Transformers are vital links in transmission and distribution system. Proper maintenance of the transformer is able to decrease maintenance personnel requirements, improve reliability and security of power supply. The condition of the transformer oil reflects the health condition of the transformer because all the energized and high temperature components are immersed in the oil. In high voltage (HV) transmission and distribution systems, transformer oil is widely used as dielectric medium in HV power apparatus such as transformer, switchgear, current transformer, potential transformer etc. It has been estimated that every year about 30 to 40 billion litres of insulating oil is used as a dielectric medium in transformers in the world [1]. Since the transformers are one of the most important power equipments in electrical systems and also the costliest, hence they need to be monitored periodically to prevent any potential fault inside the transformer.

To minimize the capital expenditure of electrical power system, it is very common to operate these equipments at their limits of design parameters. Its failures create losses of revenues and catastrophic will create substantial costs in terms of the connected equipment destruction, environmental damages and unexpected used of resources. Regular monitoring is very important in order to find the remnant life of the transformer. Under the influence of thermal and electrical stresses, oil and oil-impregnated electrical insulating materials can be decomposed and generate some hydrocarbon gases which dissolve in mineral oil. The content of dissolved decay products in insulating oils is made up of a variety of compounds, such as peroxides, aldehydes, ketenes and organic acids [2]. Each of them is partially adsorbed on the large surface of paper insulation leading to the premature aging of power transformers. Hence, these by products can be used as an indicator of the aging of the mineral oil.

UV spectrophotometer is used to describe the relative level of dissolved decay products in transformer insulating oils which are of petroleum origin. If new oil is tested then it is almost transparent to the monochromatic beam of light in the visible spectrum, the increasing concentration of dissolved decay products shift the absorbance curve to longer wavelengths as shown in Fig. 3a to 3d [3-4]. This test method is applicable to compare the extent of dissolved decay products for oils in service. There is a relationship exists between the area under the absorbance curve and the total amount of dissolved decay products in mineral insulating oils. If the curve between the absorbance and wavelengths shift to longer then it indicates an increased content of dissolved decay products in the oil, while shift of the curve between absorbance and wavelengths is shorter then it indicates the selective removal of dissolved decay products. This paper introduces two methods to determine the dissolved decay product in mineral insulating oils of power transformers for remnant life estimation mathematically by Fuzzy logic and ANN (Artificial neural Network). 


\section{Spectrophotometer Method And Oil Sampling Techniques}

An absorbance spectrophotometer measures the fraction of the incident light transmitted through a solution. It measures the amount of light that passes through a sample material and by comparison to the initial intensity of light reaching the sample, they indirectly measure the amount of light absorbed by that sample. Spectrophotometers are designed to transmit light of narrow wavelength ranges. As different compounds absorb light at different wavelengths, a spectrophotometer can be used to distinguish compounds by analyzing the pattern of wavelengths absorbed by a given sample. Additionally, the amount of light absorbed is directly proportional to the concentration of absorbing compounds in that sample, so a spectrophotometer can also be used to determine concentrations of compounds in solution. The amount of light transmitted through a solution is referred to as transmittance (T) [3].

The transmittance is defined as the ratio of the light energy transmitted through the sample (I) to the energy transmitted through the reference blank $\left(\mathrm{I}_{0}\right)$. Since the compound being tested is not present in the reference blank, the transmittance of the reference blank is defined as \% $\mathrm{T}$.

$$
\% T=\frac{I}{I_{0}} \times 100
$$

Where,

$\mathrm{T}$ is transmittance,

I is light energy transmitted through the sample,

$\mathrm{I}_{0}$ is the energy transmitted through the reference blank.

A certain portion of the light will be absorbed by the compound in the test cuvette; therefore its \% $\mathrm{T}$ will be lower than that of the blank. The Lambert-Beer law, provides a linear relationship between absorbance and concentration of an absorber of electromagnetic radiation such as [3-4]

Where,

$$
A=\varepsilon . l . c
$$

$\varepsilon=$ the extinction coefficient of the substance, has units of $\mathbf{M}^{-1} \times \mathrm{cm}^{-1}$ (unique for each substance),

$1=$ the sample path length measured in centimetres (the width of the cuvette almost always $1 \mathrm{~cm}$ ),

$\mathrm{c}=$ the molar concentration of the solution (concentration in terms of molarities),

$\mathrm{A}_{\lambda}$ also referred to as Optical Density or $\mathrm{OD}_{\lambda}$ where $\lambda$ is the wavelength used for the measurements. Absorbance is related logarithmically to transmission

$$
\begin{aligned}
& A=-\log (T) \\
& A=-\log \left(I / I_{0}\right)
\end{aligned}
$$

The absorbance of the reference blank is set at zero $\left(\mathrm{A}_{\lambda}=0\right)$. The $\lambda$ subscript is often dropped with the understanding that a value for $\varepsilon$ is for a specific wavelength. If multiple species that absorb light at a given wavelength are present in a sample, the total absorbance at that wavelength is the sum due to all substances. Absorbance is additive

$$
\begin{gathered}
A_{\text {Total }}=A_{1}+A_{2}+\ldots \ldots \ldots . . \\
A_{\text {Total }}=\varepsilon_{1} l c_{1}+\varepsilon_{2} l c_{2}+\ldots \ldots \ldots .
\end{gathered}
$$

UV spectrophotometer has been used for last 35 years and over this period has become most important analytic instrument. The UV spectrophotometer provides reasonable information on the power transformers to plan relocation and operational criteria.

\subsection{Oil samplings}

Total 12 numbers of different transformer oil samples are collected from the Himachal Pradesh Electricity Board (HPSEB), India as per ASTM STD D-6802. The transformers ratings ranges from 5-50 MVA and their voltage ratios ratings are 132/33/11 KV. The oil samples are collected on the year's basis. The transformer oil sample which are collected are as one sample of 5 MVA transformer, two samples are of 7.5 MVA transformer and three samples each of 25 MVA, 45 MVA, 50 MVA transformers. The transformer oil samples collected are from new to old or aged oil samples of last past 8 to 10 years. The data is collected from the transformers maintenance records of the operation and maintenance department. The transformers have different service periods and aging conditions. 


\section{Experimental Setup and Procedure for UV Spectrophotometer}

The experiment is done to find the dissolve decay content concentration of various samples of transformer oil. The experiment has been done according to ASTM D-6802. Initially, clean the cuvettes with the petroleum spirits or the same transformer oil sample. Adjust the UV Spectrophotometer to zero with spectral grade heptanes. This test is carried out at room temperature in the range $20^{\circ} \mathrm{C}$ to $30^{\circ} \mathrm{C}$. During this process heptane is placed in $10 \mathrm{~mm}$ path length glass cuvette, which is installed in UV spectrophotometer. The Heptane filled cuvette is then placed to the reference position in the instrument. The second glass cuvette is filled with the transformer oil sample which has to be tested [3]. The cuvette holder and cuvette containing the Heptane and transformer oil is shown in Fig.1.

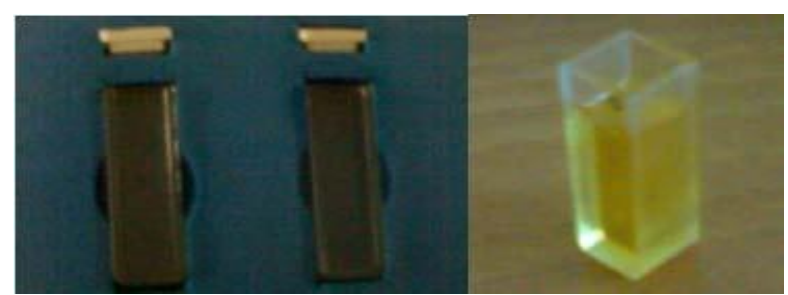

Fig.1. Cuvette holders with heptane and oil

The cuvette with oil sample is placed so that the absorbance curve of the mineral oil can be determined. The absorbance curve is obtained from the instrument which scans in the range of 360-600 $\mathrm{nm}$. The graph which is obtained is between wavelength and absorbance of the given oil samples. The relationship exists between the absorbance curve and the total amount of dissolve decay products in mineral insulating oil. The absorbance curve to the narrow wavelength indicates that the oil is new. If the curve between the absorbance and wavelengths shift to longer then it indicates an increased content of dissolved decay products in the oil, while shift of the curve between absorbance and wavelengths is shorter then it indicates the selective removal of dissolved decay products. Thus it indicates the condition of transformer oil. The complete setup of the procedure discussed above is shown in Fig. 2.



Fig.2 Setup for UV Spectrophotometer test

The assembly shows the complete setup of UV Spectrophotometer, the function of each equipment has been explained above. The graphs obtained from the UV Spectrophotometer for different oil sample are shown in Fig.3. Fig. 3(a) shows the UV spectrophotometer plot obtained from the Heptane sample which forms the base line. This is done to fulfil the zero condition of the instrument by adjusting it to read zero absorbance. The value obtained is constant and is equal to -0.092. The wavelength which the instrument is scan is in range of $360-600 \mathrm{~nm}$.

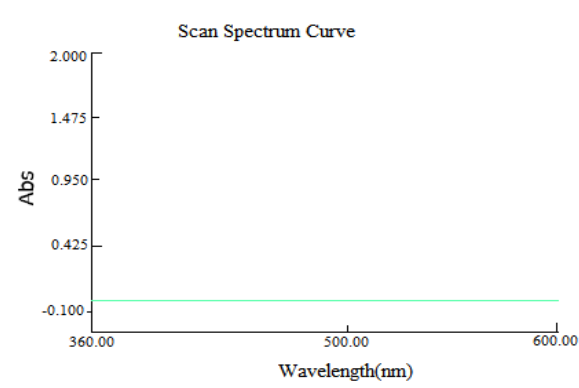

Fig.3 (a)

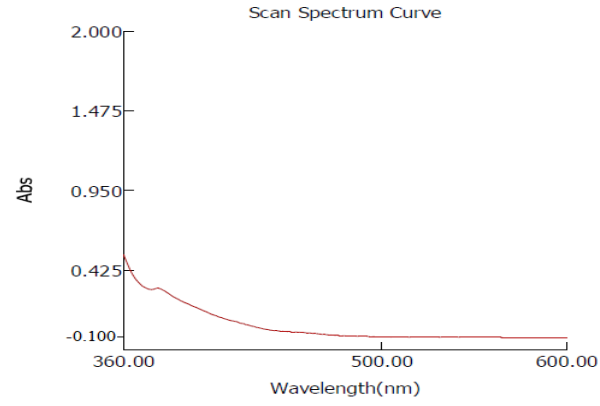

Fig.3 (b) 


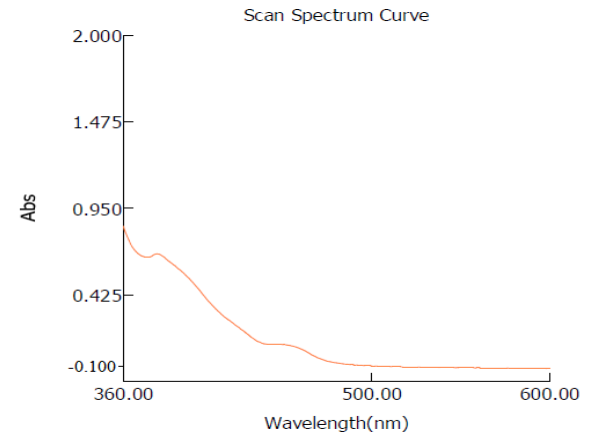

Fig.3 (c)



Fig. 3 (d)

Fig. 3. The graphs from the UV Spectrophotometer for different oil sample are (a) Show the base line (heptane) (b) Fresh transformer oil (c) Medium aged transformer oil (d) highly aged transformer oil.

Fig. 3(b) shows the UV Spectrophotometer plot obtained for fresh oil sample. For obtaining this graph the heptane-filled cuvette is moved to the reference position as mentioned previously. Now the second glass cuvette which is filled with the transformer oil sample is placed into the sample holder, and the UV scan is started. In Fig. 3(b) it is seen that there is a shift of the curve obtained from UV scan. It is seen that absorbance value is decreased from wavelength 360-600 $\mathrm{nm}$. Such behaviour of the change in the shift of the absorbance curve indicates that the oil is fresh. The UV Spectrophotometer plot obtained for medium aged transformer oil is shown in Fig. 3(c). The initial value of the absorbance has increased compared to the value in 3(b). The curve between the absorbance versus wavelength has been deviated more than the Fig.3(c), which indicates the presence of the dissolve decay products and impurities in the oil. As seen from the Fig.3 (d) that the curve between wavelength and absorbance had been increased to very high, this UV Spectrophotometer plot obtained is for highly aged transformer oil.

The absorbance curve which is obtained from this graph after UV scan indicates that there is increased content of dissolved decay products in transformer oil. An overall observation of all the plots obtained from the UV spectrophotometer shows that the curve shifts from the lower to higher side if there is a presence of impurities in the sample oil. The efficiency of the method will be high if it is possible to automatically identify the age of the oil. It will be more useful to the technicians if the information about the age of the oil is identified automatically. Hence, the decisive output regarding age of the oil in terms of characteristics like new oil, medium aged oil and highly aged oil is more useful [5].

For designing an automatic system the parameters obtained from the UV Spectrophotometer scan can be used. The age of the oil is classified as new, medium and highly aged oil. If the oil which is not in commission or of about 1 year age and if the dissolve decay content is very low then it is categorized as new oil. The oil which is tested about 5 to 7 years and if the dissolve decay content is normal to medium then it is categorized as medium aged oil. Finally, the oil sample tested after 9 to 10 years or more and if dissolve decay contents are very high then it is categorized as highly aged and it is required to be monitored. The aging depends on their contamination in the oil not on the basics of years.

\section{Transformers Life Estimation Techniques}

In the present work two techniques viz. Fuzzy logic and ANN based techniques are proposed to determine the remnant life of the transformers by determining the dissolve decay contents present in the transformer oil. The details of the techniques used are as given below and there results are discussed in later section.

\subsection{Fuzzy Logic Model}

In this section, fuzzy logic model is developed to estimate the correlation of dissolve decay contents in transformer oil and its spectral response parameters. Results of the fuzzy logic model will be then compared to the results of ANN model. Input variables for the model are the values of bandwidth wavelength and the maximum absorption for different oil samples collected from the test set-up shown in Fig.1. The output from the model is the estimated dissolve decay content in ppm (parts per million). The model is built using the graphical user interface tool provided by MATLAB [6]. Each input was fuzzified into five sets (low to extensive) [7] of Gaussian combination membership function (MF) governed by the following equation

$$
f(x, c, s, m)=\exp \left[\frac{1}{2}\left|\frac{x-c}{s}\right|^{m}\right]
$$


Where $\mathrm{c}$ is centre, $\mathrm{s}$ is width and $\mathrm{m}$ is Fuzzification factor. The function set developed for each input is a combination of $\mathrm{s}$ and c parameters. The corresponding Fuzzy logic simulation model developed is shown in Fig.4. The corresponding Gaussian function curves for each input variable are shown in Fig.5. And the corresponding Gaussian function curve for output variable is shown in Fig.6. Result of fuzzification from each input was then applied with fuzzy operator in the antecedent and related to the consequence, by IF- AND -THEN application method [8-9]. Centre of gravity which is widely used in fuzzy models was used for defuzzification method where the desired output $\mathrm{z}_{0}$ is calculated as

$$
z_{0}=\frac{\int z \cdot \mu_{c}(z) d z}{\int \mu_{c}(z) d z}
$$

Where $\mathrm{m}_{\mathrm{c}}(\mathrm{z})$ is the MF of the output.

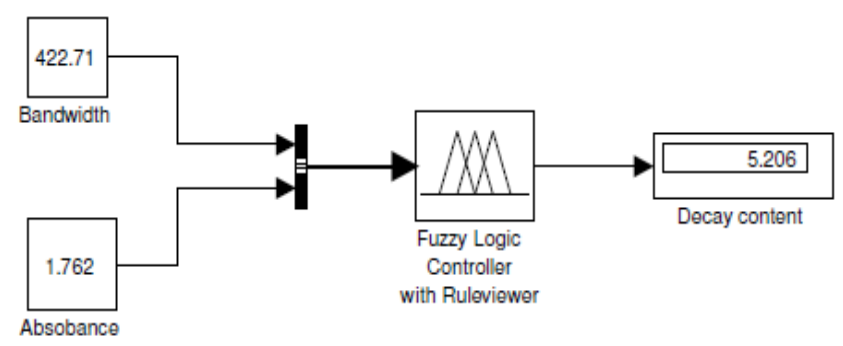

Fig.4 Fuzzy logic model for decay content estimation

The Fuzzy logic model developed using matlab simulation toolbox to estimate the remnant life of the power transformer by determining the dissolve decay contents in the transformer oil is shown in fig. 4 . It uses the Bandwidth and Absorbance values as the input and the dissolve decay content in ppm (parts per million) as the output. The model developed can be used for any input and output values which are in their ranges and it can be extended as desired.
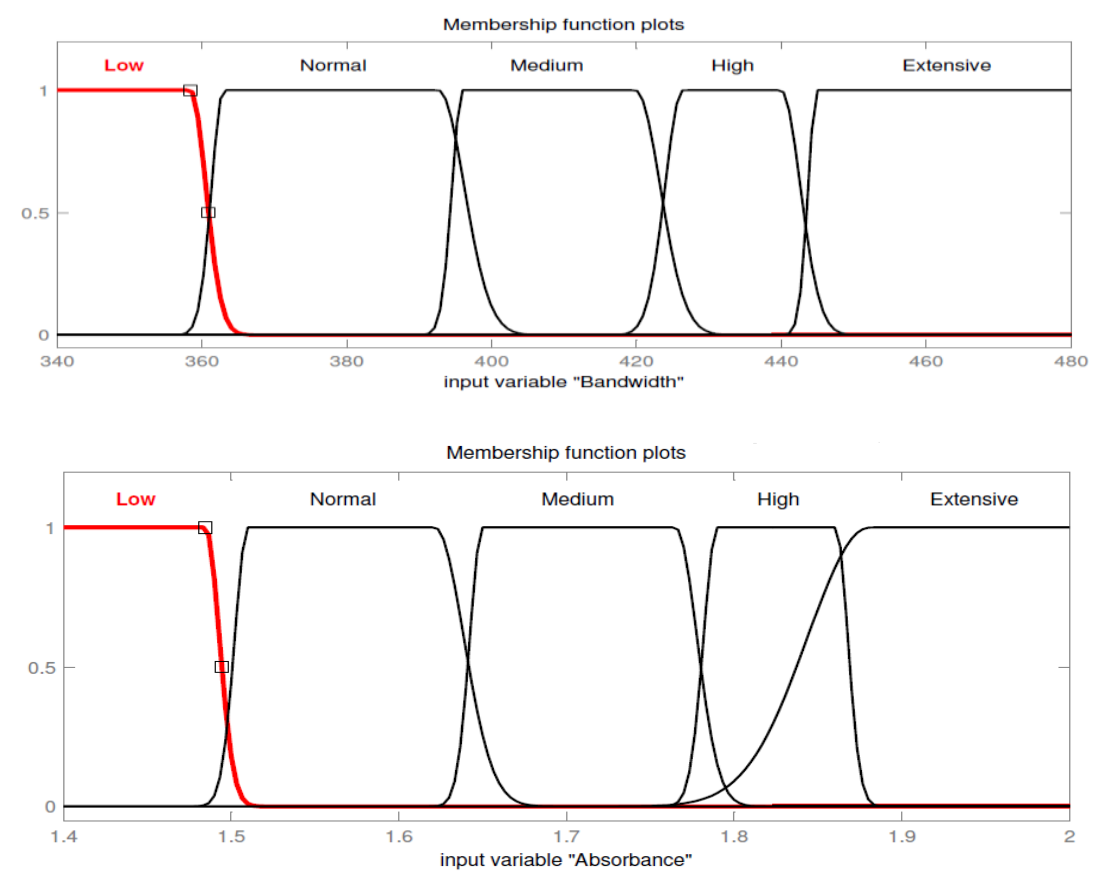

Fig.5 Input variable Membership Functions (bandwidth and absorbance)

The input membership functions for Bandwidth and Absorbance is shown in fig.5 and the membership functions (MF) used is the Gaussian MF function. The function is divided in five sets as low, normal, medium, 
high and extensive. Bandwidth wavelength ranges from $340 \mathrm{~nm}$ to $480 \mathrm{~nm}$ and Absorbance ranges from $1.4 \mathrm{~nm}$ to $2 \mathrm{~nm}$.

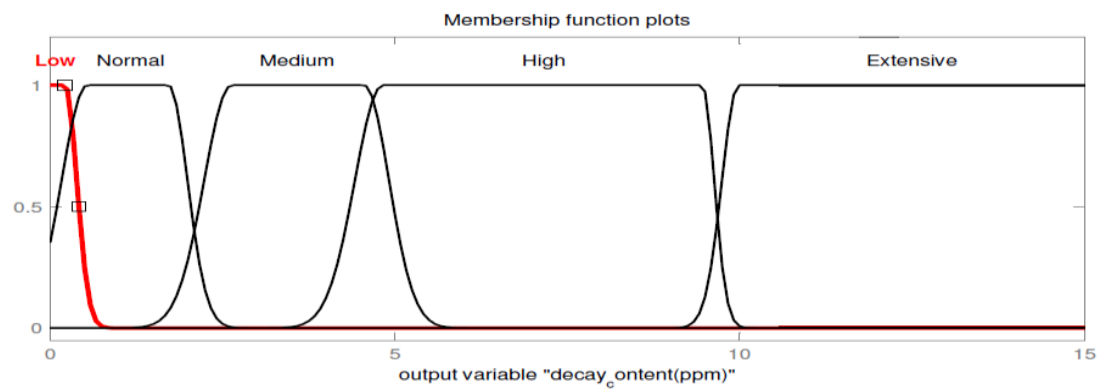

F ig.6 Output variable Membership Function (decay content)

The output variable membership functions is shown in Fig.6. The MF used is Gaussian MF and is fuzzified in five sets as that of the inputs. The output is dissolve decay content which ranges from $0 \mathrm{ppm}$ to $15 \mathrm{ppm}$.

\subsection{Artificial Neural Network}

In this section the neural network simulation is developed using Matlab to estimate the correlation of dissolve decay content and the spectrophotometer response of the transformer oil [6]. Artificial Neural networks are a relatively new artificial intelligence technique. An artificial neural network (ANN) is determined by its architecture, training method and exciting function. Its architecture determines the pattern of connections among neurons. The neurons are connected by links and each link has a numerical weight associated with it. Weights are the basic means of long-term memory in ANNs. They express the strength or importance of each neuron input. Network training changes the values of weights and biases in each step in order to minimize the mean square of output error. Multi-Layer Perceptron (MLP) has been used in load forecasting, nonlinear control, system identification and pattern recognition [2-10], thus in this paper multi-layer perceptron network (with two inputs, one outputs and a hidden layer) with Levenberg-Marquardt training algorithm have been used.

\subsubsection{Architecture and input-output of ANN}

A multilayer perceptron is a feedforward neural network with one or more hidden layers. The input signals are propagated in a forward direction on a layer-by-layer basis. In the Fig.7 a multilayer neural network with two hidden layer has been shown [11]. The Matlab model developed was with the twenty two numbers of neurons in the hidden layer.


Fig.7 Multilayer perceptron with two hidden layers and Inputs and Output of ANN

For the neural network the input and output is required, so the spectrophotometer response with Bandwidth and Absorbance are given as input signals where as the dissolve decay content as the output signal. A typical network with input and output is shown in Fig.7.

ANN is made up of a hierarchy of layers, and the neurons in the networks are arranged along these layers. The neurons connected to the external environment form input and output layers. The weights are modified to bring the network input/output behaviour into line with that of the environment. Each neuron is an elementary information-processing unit. It has a means of computing its activation level given the inputs and numerical weights. To build an artificial neural network, we must decide first how many neurons are to be used and how the neurons are to be connected to form a network. Then we decide which learning algorithm to use and finally we train the neural network.

The back propagation training algorithm is a method of iteratively adjusting the neural network weights until the desired accuracy level is achieved. It is based on a gradient- search optimization method 
applied to an error function. The network has been trained with Levenberg-Marquard back propagation algorithm. The number of neurons in hidden layer is twenty two.

\section{Results and Discussions}

Data for the methodologies as described in section 4 are generated according to the procedure given in section 3 of the paper. A total number of 12 transformer oil samples are taken for the study purpose. Using spectrophotometer the transformer oil samples are tested and obtained results are given in Table 1 .

Table1. Results of UV Spectrophotometer

\begin{tabular}{|l|l|l|l|}
\hline $\begin{array}{l}\text { Oil } \\
\text { samples }\end{array}$ & $\begin{array}{l}\text { Bandwidth } \\
(\mathbf{n m})\end{array}$ & $\begin{array}{l}\text { Absorbance } \\
(\mathbf{n m})\end{array}$ & $\begin{array}{l}\text { Dissolve Decay } \\
\text { Contents (ppm) }\end{array}$ \\
\hline 1 & 355.23 & 1.540 & 0.500 \\
\hline 2 & 365.38 & 1.560 & 1.000 \\
\hline 3 & 382.54 & 1.642 & 2.000 \\
\hline 4 & 395.82 & 1.681 & 3.000 \\
\hline 5 & 410.33 & 1.742 & 4.000 \\
\hline 6 & 422.71 & 1.762 & 5.000 \\
\hline 7 & 433.82 & 1.789 & 7.000 \\
\hline 8 & 443.51 & 1.821 & 10.000 \\
\hline 9 & 450.21 & 1.850 & 11.000 \\
\hline 10 & 456.23 & 1.873 & 12.000 \\
\hline 11 & 463.25 & 1.932 & 13.000 \\
\hline 12 & 473.81 & 1.961 & 15.000 \\
\hline
\end{tabular}

The dissolve decay content is in the range $0 \mathrm{ppm}$ to $15 \mathrm{ppm}$. When the dissolve decay content for the transformer oil is reached to more than 12ppm that means its life has reduced and it needs to be maintained. The Fuzzy logic model which is developed using Matlab graphical user inference is with the accuracy of more than $95 \%$. The ANN network model which is trained using Levenberg-Marquardt back propagation algorithm was able to correlate with dissolve decay content with accuracy more than $99 \%$. The results obtained from the Fuzzy logic model and ANN model are compared with Spectrophotometer results which is shown in Table 2. The fuzzy logic model results are given in column 3 and ANN results are given in column 4 of Table 2.

Table2. Comparison of Fuzzy and ANN model with UV Spectrophotometer results

\begin{tabular}{|l|l|l|l|l|}
\hline $\begin{array}{l}\text { Oil } \\
\text { Samples }\end{array}$ & Target & $\begin{array}{l}\text { Fuzzy Model } \\
\text { output }\end{array}$ & $\begin{array}{l}\text { ANN model } \\
\text { estimated output }\end{array}$ & $\begin{array}{l}\text { ANN Model } \\
\text { Absolute Error }\end{array}$ \\
\hline 1 & 0.500 & 0.98 & 0.496 & 0.004 \\
\hline 2 & 1.000 & 1.09 & 0.994 & 0.006 \\
\hline 3 & 2.000 & 2.48 & 2.000 & 0.000 \\
\hline 4 & 3.000 & 3.57 & 3.002 & 0.002 \\
\hline 5 & 4.000 & 3.60 & 4.001 & 0.001 \\
\hline 6 & 5.000 & 5.21 & 5.000 & 0.000 \\
\hline 7 & 7.000 & 7.22 & 7.000 & 0.000 \\
\hline 8 & 10.000 & 9.80 & 10.000 & 0.000 \\
\hline 9 & 11.000 & 12.30 & 11.000 & 0.000 \\
\hline 10 & 12.000 & 12.40 & 11.998 & 0.002 \\
\hline 11 & 13.000 & 13.58 & 13.000 & 0.000 \\
\hline 12 & 15.000 & 13.78 & 14.890 & 0.110 \\
\hline
\end{tabular}

The ANN model Absolute Error is found by following formula

$$
\text { Absolute Error }=\mid \text { ANN output - Target } \mid
$$

The Fuzzy logic results are graphically shown in Fig.8 and Fig.9. The variation range in wavelength and absorbance and the corresponding decay content concentration are used to develop a set of fuzzy logic rules in the form of (IF-AND-THEN) statements to relate the input variables to the output, the rules are shown in Fig.8. The number of rules developed are 25 and the corresponding three dimensional graph obtained from Matlab is shown in Fig.9. The results which obtained using Fuzzy logic are with accuracy more than $95 \%$. The examples of the rules developed are as follow.

1. If Bandwidth is low and Absorbance is low then decay content is low.

2. If Bandwidth is normal and Absorbance is normal then decay content is normal.

3. If Bandwidth is medium and Absorbance is medium then decay content is medium.

4. If Bandwidth is high and Absorbance is high then decay content is high and so on. 
In the Fig. 8 the Bandwidth is $423 \mathrm{~nm}$ and Absorbance is $1.76 \mathrm{~nm}$ then the corresponding decay content is $5.21 \mathrm{ppm}$. The Fuzzy logic results and spectrophotometer results are compared in Table 2. The three dimensional graph in Fig.9 shows the variation of the Bandwidth to Absorbance and Decay contents. It shows the transformer oil samples different aging. From blue to yellow the graph shows that when the Absorbance of the transformer oil starts increasing the dissolve decay contents are increased accordingly and shows the aging of the transformer.
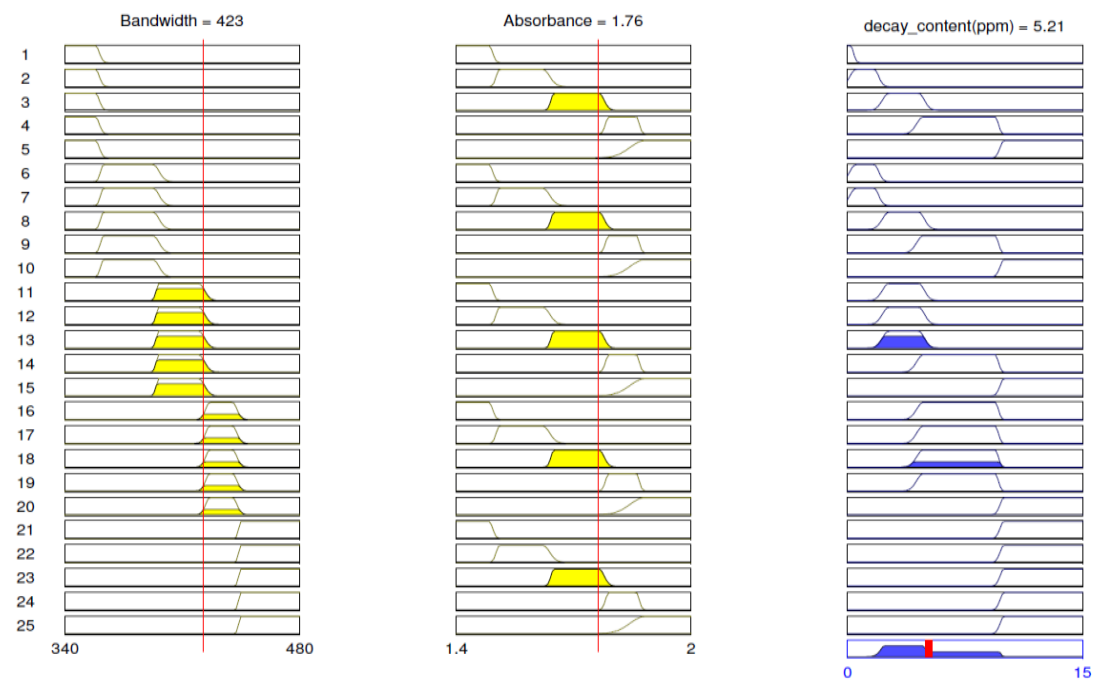

Fig.8 Fuzzy rules-Spectrophotometer decay content model

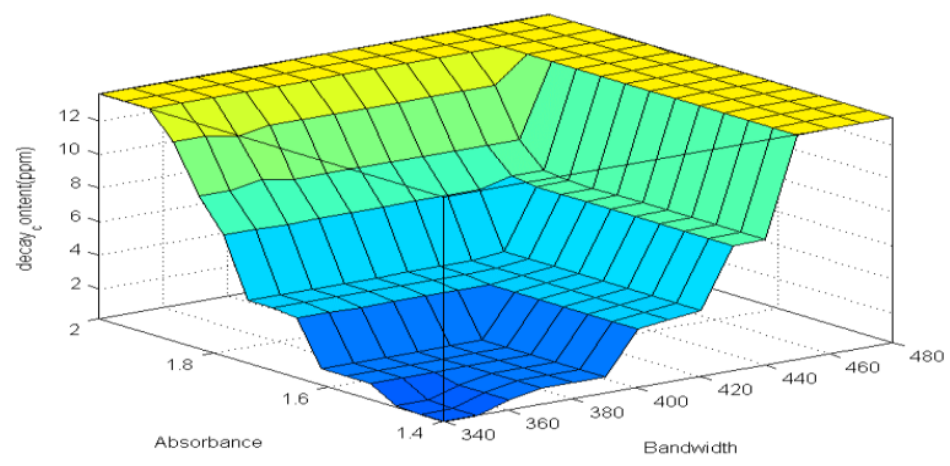

Fig. 9 Three dimensional mapping from bandwidth and absorption peak to decay content

The ANN simulation results are graphically shown in Fig. 10 to Fig13. The performance curve is shown in Fig.10. Mean Squared Error is the average squared difference between outputs and targets. Lower values are better. Zero means no error. In this figure mean squared error have become small by increasing the number of epoch. The best validation performance is 1.0054 at epoch 5. It means that target has tracked the output very efficiently.

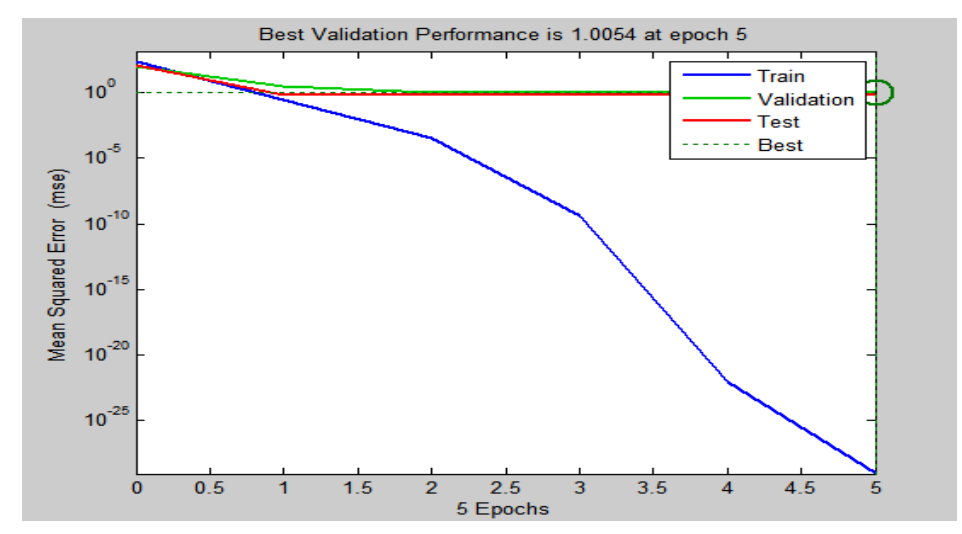


Fig.10 Mean Square Error of ANN model

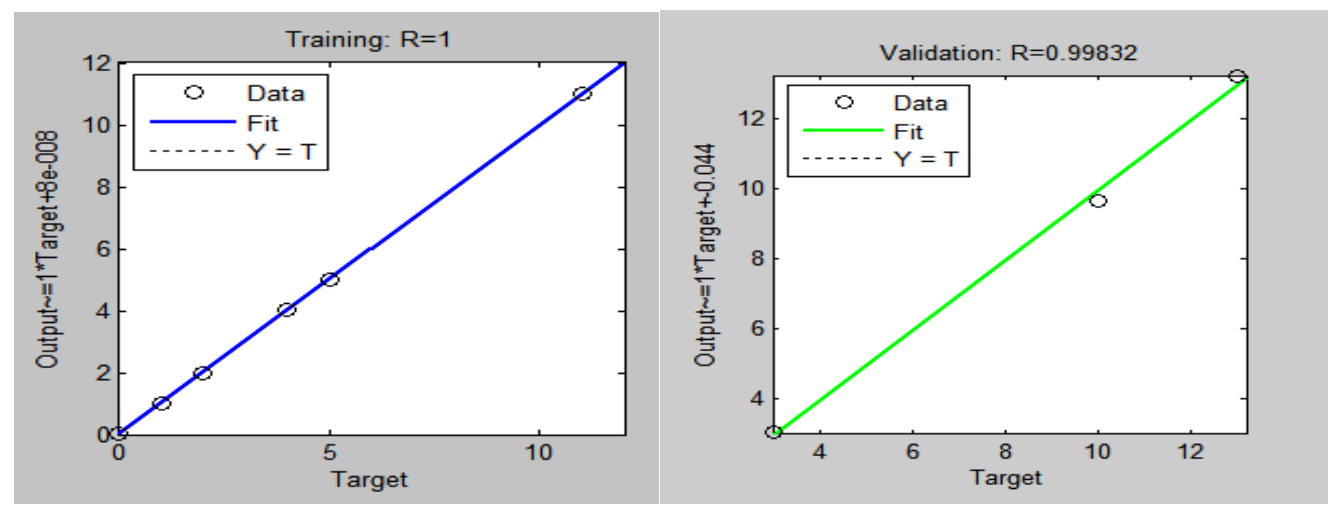

Fig.11 Prediction of Dissolve decay contents during training and validation analysis

During training of the output dissolve decay content is shown in Fig.11. These data are presented to the network during training, and the network is adjusted according to its error. As it can be seen that regression is equal to one which indicates close relation between target and output. The validations are used to measure network generalization, and to halt training when generalization stops improving. The validation regression plot is shown in Fig. 11 and value of $\mathrm{R}$ is over 0.99 .
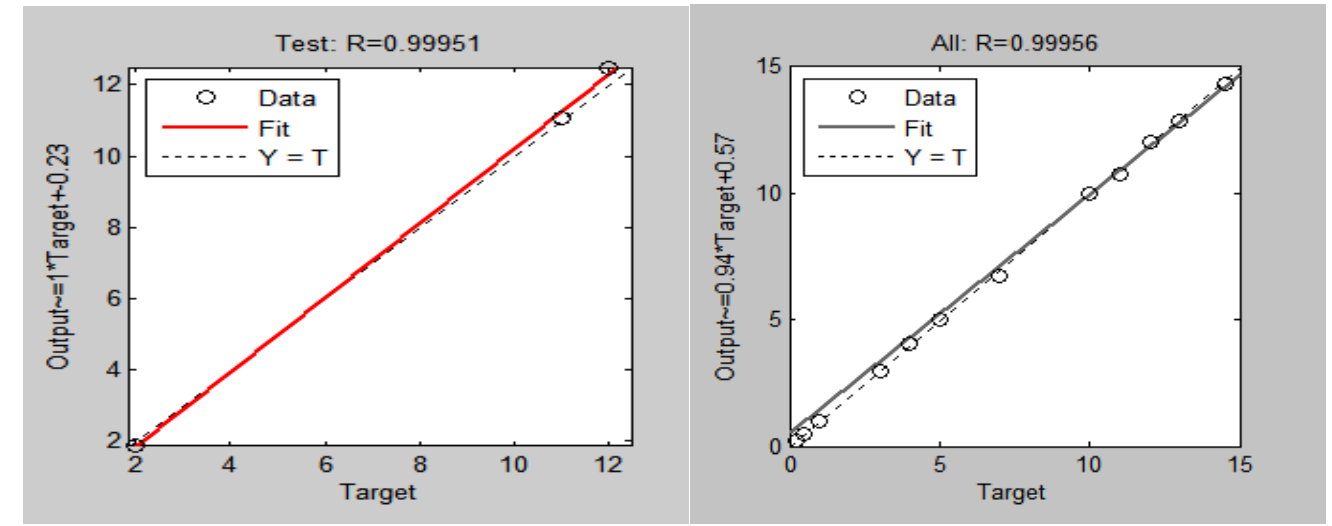

Fig.12 Prediction of Dissolve decay contents during testing analysis and during regression

The testing plot is shown in Fig.12. These have no effect on training and it provides an independent measure of network performance during and after training and the value of Regression $\mathrm{R}$ is 0.99951 . The Regression plot is shown in Fig. 12 its values measure the correlation between outputs and targets. If the value of $\mathrm{R}$ is 1 means a close relationship between target and output and if value of $\mathrm{R}$ is 0 then there is a random relationship between target and output of ANN.

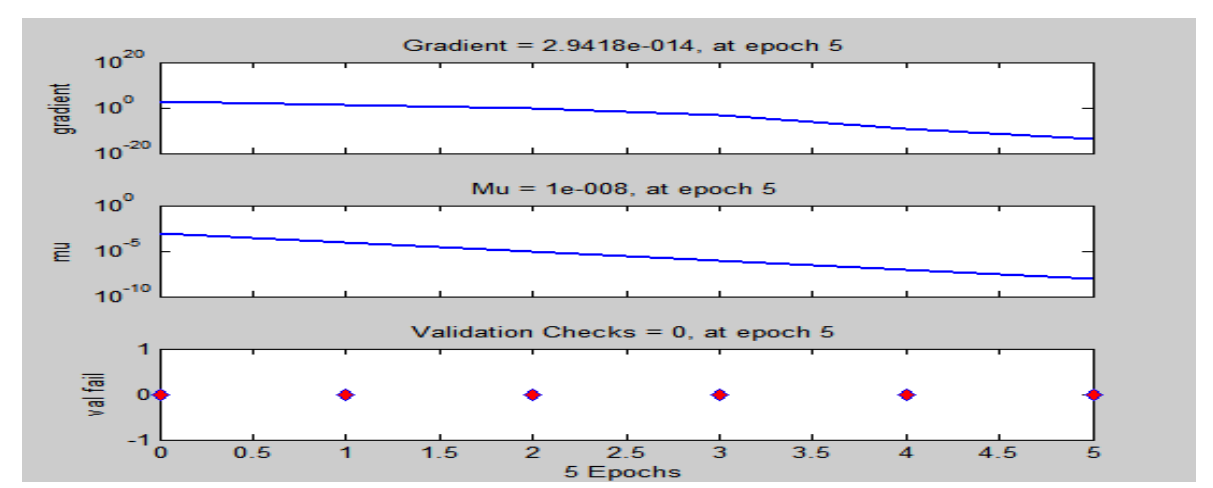

Fig.13 Training state plot for decay content estimation in transformer oil samples

The training state plot for dissolve decay content estimation is shown in Fig.13, it is seen that the training, validation, testing and regression plots all are over 0.999 . Which shows a good response between the 
output and targets which are selected. Hence, it is seen that the output of the ANN has tracked the dissolve decay content very well.

\section{Conclusion}

Power transformers are very important in the transmission and distribution system. Hence the monitoring and diagnosis is very important for it. In this paper the Spectrophotometer method is used to detect the dissolve decay contents in transformer oil for replacement of the transformer oil. The UV spectrophotometer response of transformer oil can be measured instantly using relatively cheap equipment with no need to an expert person to perform the test. Results show that there is a relationship between dissolve decay content in transformer oil and its spectral response parameters with bandwidth and absorption. The paper introduces a novel fuzzy logic and ANN approach to estimate the transformer expected remnant life and used to find this relationship mathematically. It is found from comparison that all the results obtained from Fuzzy logic is almost equal with an accuracy of over $95 \%$ and ANN with an accuracy more than $99 \%$ as obtained from the UV spectrophotometer.

\section{Acknowledgements}

The authors are thankful to the Technology Information Forecasting and Assessment Council and Centres of Relevance \& Excellence (TIFAC-CORE) at NIT Hamirpur for providing the necessary facilities to perform the research work. And the authors are also thankful to the Himachal Pradesh Electricity Board (HPSEB), India for providing the transformer oil samples for experiment.

\section{References}

[1] T. K. Saha, "Review of Modern Diagnostic techniques for Assessing Insulation Condition Aged Transformers," IEEE Transaction on Dielectrics and Electrical Insulation, vol. 10, pp. 903-917, 2003.

[2] Hasmat Malik, A.Z and S.M, "Artificial Intelligence Techniques for Incipient Faults Diagnosis and Condition Assessment in Transformer" in Proc. Inter. Conf. on Emerging Trends in Engineering ISBN 978-93-81195-07-9, 2011, pp.5-8.

[3] ASTM D6802-02 (Reapproved 2010) standards; "Test Method for Determination of the Relative Content of Dissolved Decay Products in Mineral Insulating Oils by Spectrophotometry".

[4] Manual T90/T90+ "UV/VIS Spectrophotometer" [PG Instrument] TIFAC-CORE "NIT-HAMIRPUR.

[5] U. Mohan Rao, A.Pramoda \& D.Vijay Kumar, "Decision Tree Based UV/VIS Response To Access the age of Transformer Oil" International Journal of Power System Operation and Energy Management, ISSN (PRINT): 2231-4407, Volume-1, Issue-2, 2011.

[6] MATLAB R2009b version 7.9.0.529.

[7] A. Abu-Siada, Lai Sin Pin, and Syed Islam, "Remnant Life Estimation of Power Transformer using Oil UV Vis Spectral Responses" Paper accepted for presentation at IEEE PSCE 09 that held in USA, March, 2009.

[8] S. M. Islam, T. Wu, G. Ledwich, “A Novel Fuzzy Logic Approach to Transformer Fault Diagnosis," IEEE Transaction on Dielectric and Electrical Insulation, Vol.7 No. 2,April 2000, pp. 177-186.

[9] A.Abu-Siada, M. Arshad, and S. Islam, "Fuzzy Logic Approach to Identify Transformer Criticality using Dissolved Gas Analysis," 978-1-4244-65514/10/@2010 IEEE.

[10] Hasmat Malik, S.M, R.K. Jarial, YRS, "Application and Implementation of Artificial Intelligence in Electrical System" published in international conference on Advances in Computing \& Communication (ICACC-2011) ISBN 978-81-920874-0-5, Sponsored by IEEE-MTTS, pp. 499-505.

[11] Michael Negnevitsky “Artificial Intelligence A Guide to Intelligent Systems Second” Pearson Education Limited 2002.



Dr.Ashwani Kumar Chandel graduated in Electrical Engineering from Kerala University. He post graduated from Punjab Engineering College (PEC) Chandigarh. He was awarded Ph.D. degree from Indian Institute of Technology (IIT), Roorkee, India in 2005. Dr. Kumar joined Department of Electrical

Engineering, National Institute of Technology, Hamirpur, HP, India, as faculty in 1991, where presently he is working as an Associate Professor and networking coordinator. His research work has been published in various International Journals of repute including IEEE, IEE, Elsevier Science, Taylor \& Francis and others. He has worked extensively in the area of harmonic estimation \& elimination and currently his interest continues in this field. He is a life member of ISTE (I).

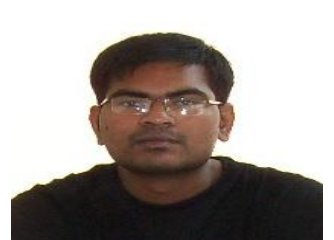

Satyaprakash Ram graduated in Electrical Engineering from Government College Of Engineering Chandrapur, Maharashtra in the year 2009. He is currently pursuing his M.Tech in Condition Monitoring Control and Protection of Electrical Apparatus from NIT Hamirpur, India. He has worked extensively in the area of the condition monitoring of the transformer.

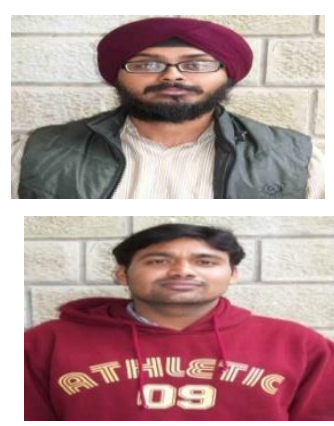

Gurmeet Singh graduated in Electrical and Electronics Engineering from College Of Engineering Roorkee in the year 2009. He is currently pursuing his M.Tech in Condition Monitoring Control and Protection of Electrical Apparatus from NIT Hamirpur, India.

Mithun Mondal graduated in Electrical and Electronics Engineering from Dr. M.G.R Engineering College, Chennai in the year 2009. He is currently pursuing his M.Tech in Condition Monitoring Control and Protection of Electrical Apparatus from NIT Hamirpur, India. 